\title{
Protocol
}

\section{Cloning Mice}

\author{
Atsuo Ogura
}

Viable and fertile mice can be generated by somatic nuclear transfer into enucleated oocytes, presumably because the transplanted somatic cell genome becomes reprogrammed by factors in the oocyte. The first somatic cloned offspring of mice were obtained by directly injecting donor nuclei into recipient enucleated oocytes. When this method is used (the so-called Honolulu method of somatic cell nuclear transfer [SCNT]), the donor nuclei readily and completely condense within the enucleated metaphase II-arrested oocytes, which contain high levels of M-phase-promoting factor (MPF). It is believed that the condensation of the donor chromosomes promotes complete reprogramming of the donor genome within the mouse oocytes. Another key to the success of mouse cloning is the use of blunt micropipettes attached to a piezo impact-driving micromanipulation device. This system saves a significant amount of time during the micromanipulation of oocytes and thus minimizes the loss of oocyte viability in vitro. For example, a group of 20 oocytes can be enucleated within 10 min by an experienced operator. This protocol is composed of seven parts: (1) preparing micropipettes, (2) setting up the enucleation and injection micropipettes, (3) collecting and enucleating oocytes, (4) preparing nucleus donor cells, (5) injecting donor nuclei, (6) activating embryos and culturing, and (7) transferring cloned embryos.

It is essential that you consult the appropriate Material Safety Data Sheets and your institution's Environmental Health and Safety Office for proper handling of equipment and hazardous material used in this protocol.

RECIPES: Please see the end of this protocol for recipes indicated by $<R>$. Additional recipes can be found online at http://cshprotocols.cshlp.org/site/recipes.

Reagents

Cytochalasin B (Sigma-Aldrich C6762)

$\mathrm{CZB}, \mathrm{CZB}-\mathrm{HEPES}$, and CZB-Sr media for mouse cloning $<\mathrm{R}>$

Human chorionic gonadotropin (hCG)

Hyaluronidase (0.3-1 mg/mL solution in CZB medium) (e.g., Sigma-Aldrich H3884)

Mature female mice ( $8-12 \mathrm{wk}$ of age)

See Box 1.

Mercury or Perfluoro-compound FC-77 (Fluorinert, ACROS Organics 123810250, or Sigma-Aldrich F3556)

Polyvinylpyrrolidone (PVP; 10\%-12\%; $360 \mathrm{kDa})<\mathrm{R}>$

Pregnant mare serum gonadotropin (PMSG)

From the Manipulating the Mouse Embryo collection, edited by Richard Behringer, Marina Gertsenstein, Kristina Vintersten Nagy, and Andras Nagy.

(C) 2017 Cold Spring Harbor Laboratory Press

Cite this protocol as Cold Spring Harb Protoc; doi:10.1101/pdb.prot094425 
A. Ogura

\section{BOX 1. STRAIN CHARACTERISTICS OF RECIPIENT OOCYTES}

The strain of mouse from which recipient oocytes are collected is selected on the basis of developmental ability, tolerance to in vitro handling (in particular, intracytoplasmic injection), and visibility of metaphase II (MII) chromosomes of the oocytes. B6D2F1 (C57BL/6×DBA/2) oocytes are the best recipients and fill all of these requirements. Because oocytes from other strains may also offer these advantages, researchers can select the strain according to their purposes.

The following is a brief guide to the relevant strain characteristics.

1. High developmental ability: B6D2F1, B6C3HF1, B6CBAF1, and probably other $\mathrm{F}_{1}$ hybrids

2. Tolerance to intracytoplasmic injection: B6D2F1 and DBA/2 (best)

3. Visibility of MII chromosomes: B6D2F1, DBA/2, ICR, and 129

Silicone oil (e.g., Sigma-Aldrich 14615-3) or mineral oil (Nakalai Tesque 26132-35)

The embryo-tested oil is used to overlay the drops of culture medium. Oocytes and embryos are cultured in $5 \%-6.5 \% \mathrm{CO}_{2}$ in air at $37^{\circ} \mathrm{C}-37.5^{\circ} \mathrm{C}$.

Strontium chloride (Sigma-Aldrich S0390)

Equipment

Capillary tubes, plastic

Capillary tubing, glass (Sutter Instrument Company B100-75-10 or Drummond Microcaps [50 $\mu \mathrm{L}$, 1000-0500)

Incubator (humidified; $37^{\circ} \mathrm{C} 5 \% \mathrm{CO}_{2}$ )

Injectors (Narishige, Eppendorf International, or Leica)

To increase the survival rate of oocytes after injection, the injection micropipette must move precisely along the $x$-axis without any wavering, especially when the pipette is being withdrawn from the ooplasm. For this reason, three-dimensional (3D) micromanipulators with separate $x$-and $y$-axis handles are recommended (e.g., Narishige MO-202U joystick micromanipulators, the large-handled type) (Fig. 1). Microinjectors for injection and enucleation must be the oil-filled type rather than the air-filled type; Narishige IM-6 and IM-9 and Eppendorf International CellTram Oil work best. Injectors that are connected to the holding pipette can be the air-filled type.

Inverted microscope (Nikon, Olympus, or Leica) with Nomarski or Hoffman optics

Microdrop culture dish

Microforge (Narishige or De Fonbrune)

Micromanipulation chamber (see Step 6)

Micromanipulators (Narishige or Leica)

Petri dishes (35, 50, and $60 \mathrm{~mm}$ ) (e.g., BD Falcon 35-1008, 35-1006, 35-1007), plastic

Piezo impact-driven system (Prime Tech Ltd.)

Pipette puller (Sutter Instrument Company)

Syringe $(1 \mathrm{~mL})$

METHOD

This protocol contains some modifications of the protocols originally developed by Wakayama et al. (1998).

Part 1: Preparing Micropipettes

For efficient enucleation and injection with a piezo-driven micromanipulation device (Fig. 2), the wall of the enucleation and injection pipettes must be thin (less than one-fourth the size of the radius). The tips of the pipettes must be blunt. Using tapered tips often results in low oocyte-survival rates after enucleation or injection.

1. Break the tips of the pipettes vertically using a microforge to create a blunt end (see Fig. $1 \mathrm{~A}$ in Protocol: Pronuclear Transplantation in the Mouse Embryo [McGrath et al. 2017]). 


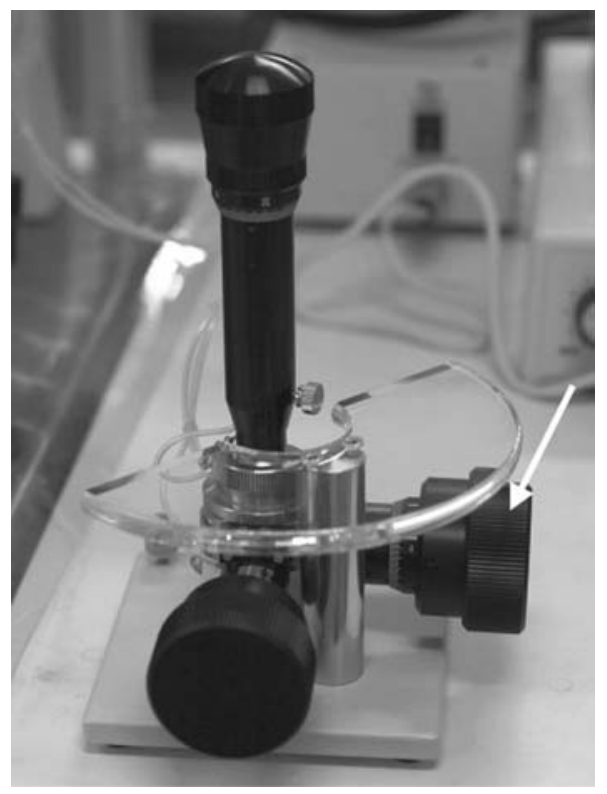

FIGURE 1. Joystick of a typical 3D micromanipulator (Narishige MO-202U). The $x$-axis handle (arrow) for fine movement is necessary for efficient operation of the piezo-driven micromanipulator.

Blunt-ended pipettes are able to break the zona pellucida and oolemma but cause minimal damage to the oocyte. The inner diameter of the enucleation pipettes is between 7 and $8 \mu \mathrm{m}$.

It is possible to thin the wall of the capillary with hydrofluoric acid, which may slightly improve the oocyte survival rate, but it is not necessary.

2. Adjust the inner diameter of the injection micropipettes according to the size and hardness of the donor cells.

Inner diameters of 4-5 $\mu \mathrm{m}$ for cumulus cells and primordial germ cells, 3-4 $\mu \mathrm{m}$ for immature Sertoli cells, and 7-9 $\mu \mathrm{m}$ for fibroblast cells are recommended.

3. Prepare holding pipettes the same way as for other mouse embryo manipulations (see Protocol: Making Holding Pipettes [Nagy et al. 2006a]).

\section{Part 2: Setting Up the Enucleation and Injection Micropipettes}

This step has a significant effect on the efficiency of nuclear transfer. If the setup is performed correctly and carefully, 100-150 oocytes can be injected before the injection pipette needs to be changed.

There are two reagents that can be used to stabilize piezo-driven microcapillaries. Mercury is the traditional choice, although it is toxic and requires specialized handling and disposal. A newer alternative is perfluoro compounds, such

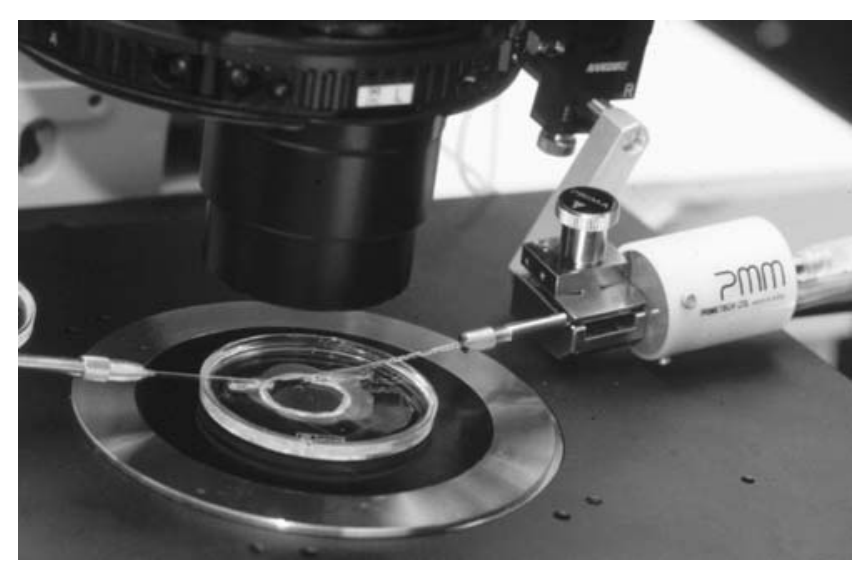

FIGURE 2. Impact unit of the piezo-driven micromanipulator system (right) attached to an injection pipette holder on an inverted microscope. 
as Fluorinert, also called FC-77. Fluorinert is chemically stable, nonflammable, and considered nontoxic, although it can cause eye, skin, and respiratory irritation. Both options are described here; perform either Step 4 or Step 5.

4. Use mercury when setting up the micropipettes.

i. Load a small quantity of mercury (1-2 mm long when in the micropipette) into the enucleation/injection micropipette from the proximal end using a small, flexible plastic capillary tube attached to a $1-\mathrm{mL}$ syringe.

ii. Attach the enucleation/injection micropipette that is connected to the oil-filled injector to the instrument holder of the piezo impact drive. After expelling the air from the tip of the enucleation/injection micropipette, wash the inside of the tip several times with oil while it is in the micromanipulation chamber (Fig. 3A).

iii. Wash the enucleation/injection micropipette in PVP medium until the mercury inside the pipette can be moved smoothly and without any time lag (Fig. 3B).

Take care to completely remove any oil adhering to the inside of the pipette.

iv. Place a small volume of oil between the PVP and the CZB-HEPES medium to prevent the mercury from contaminating the micromanipulation medium (Fig. 3C).

v. Proceed to Step 6.

5. Use Fluorinert when setting up the micropipettes.

A
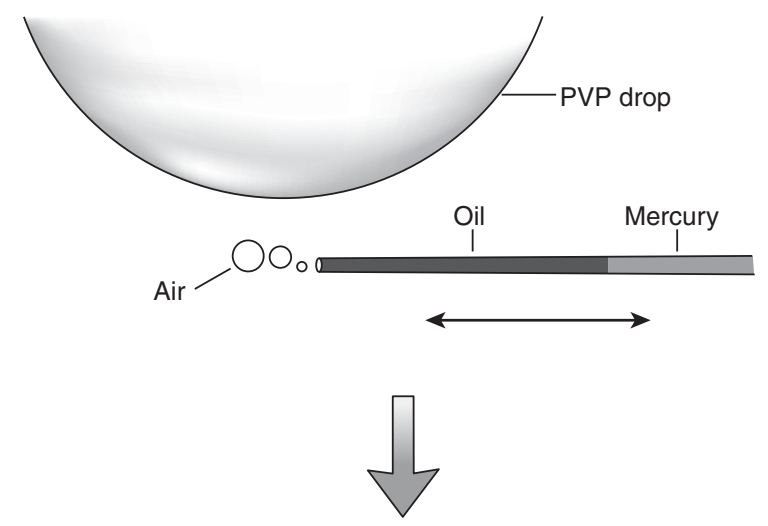

B
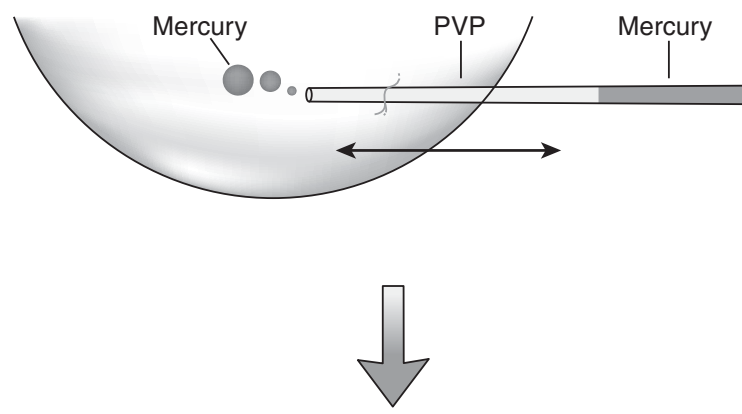

C

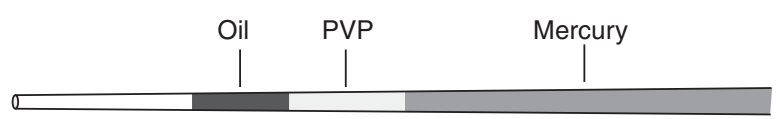

FIGURE 3. Setting up an enucleation pipette. The procedure is the same for injection pipettes (see text for details). Note that the PVP drop and pipette are under oil. (A) Air is expelled from the tip of the enucleation/injection micropipette; the tip is washed several times with oil and then with PVP medium $(B)$ until the mercury is moved smoothly inside the pipette. (C) A small volume of oil is placed between the PVP and the CZB-HEPES medium to prevent the mercury from contaminating the micromanipulation medium. 
Part 3: Collecting and Enucleating Oocytes

8. Inject mature female mice intraperitoneally with $7.5 \mathrm{IU}$ of PMSG. Let the mice rest for $48 \mathrm{~h}$. Inject them with 7.5 IU of hCG.

9. Collect mature oocytes that are surrounded by cumulus cells from the ampullae of the oviducts 15-17 h after the hCG injection.

Oocytes retrieved from the oviducts sooner than recommended (e.g., 13 h) after hCG treatment generally show better developmental ability but are less tolerant to intracytoplasmic injection.

10. Place the collected oocytes in CZB medium containing $0.1 \%$ hyaluronidase, and then place them in a $37^{\circ} \mathrm{C} 5 \% \mathrm{CO}_{2}$ humidified incubator until the cumulus cells disperse (2-3 min).

11. Wash the oocytes, and place them in a microdrop culture dish in the incubator.

12. Place a group of 10-20 oocytes into a drop of CZB-HEPES medium containing cytochalasin B in a micromanipulation chamber.

13. Hold the oocytes with the metaphase plate between the 2 o'clock and 4 o'clock positions.

14. Place the tip of the enucleation pipette onto the surface of the zona pellucida.

15. Use a few piezo pulses (controller settings: speed 3-6, intensity 1-4) to advance the pipette while applying very slight negative pressure.

To avoid damaging the oolemma, apply slight positive pressure when the perivitelline space narrows under the impacted area just before the entire zona is pierced.

16. Remove the chromosomes by suction, along with a small volume of cytoplasm.

Warming the microscope stage is recommended because the spindle microtubules will remain well polymerized and form a small clear area that can be easily distinguished from the surrounding opaque cytoplasm.

Metaphase II chromosomes are visible without the use of fluorescent dye under Nomarski or Hoffman optics. Oocytes with a clear cytoplasm (such as B6D2F1 oocytes; see above) can be more easily enucleated than those with granulated masses (such as B6 and B6CBAF1 oocytes).

17. After a group of oocytes has been enucleated, wash the oocytes several times in drops of fresh CZB medium.

18. Leave the oocytes in the incubator for $30 \mathrm{~min}$ to $2 \mathrm{~h}$ before donor nucleus injection or electrofusion.

Oocytes are very sensitive and can easily lyse just after enucleation. However, their viability is usually restored after they have been incubated as described. 
A. Ogura

Part 4: Preparing Nucleus Donor Cells

19. Collect cumulus cells from freshly ovulated donor oocytes by treatment with $0.1 \%$ bovine testicular hyaluronidase (Sigma-Aldrich H3884) in CZB medium, as described above in Part 3: Collecting and Enucleating Oocytes (and Protocol: Collecting Zygotes and Removing Cumulus Cells with Hyaluronidase [Nagy et al. 2006b]).

\section{Part 5: Injecting Donor Nuclei}

In the original trials of intracytoplasmic injection of mouse oocytes, the micromanipulation temperature was maintained at $17^{\circ} \mathrm{C}-18^{\circ} \mathrm{C}$ to enhance the survival rate. However, this is not necessary, and the operation can be performed at room temperature without any problems. A heated stage may cause adverse effects such as oocyte lysis.

20. Gently mix the donor cumulus cells into the PVP drops in the micromanipulation chamber.

Use cumulus cells from an $F_{1}$ hybrid for initial mouse cloning attempts.

21. Place a group of 10-20 enucleated oocytes in a drop of CZB-HEPES in a micromanipulation chamber.

22. Remove the nuclei from the donor cells by gently aspirating them in and out of the injection pipette. Then, draw up the donor nuclei in a line within the injection pipette (Fig. 4A,B).
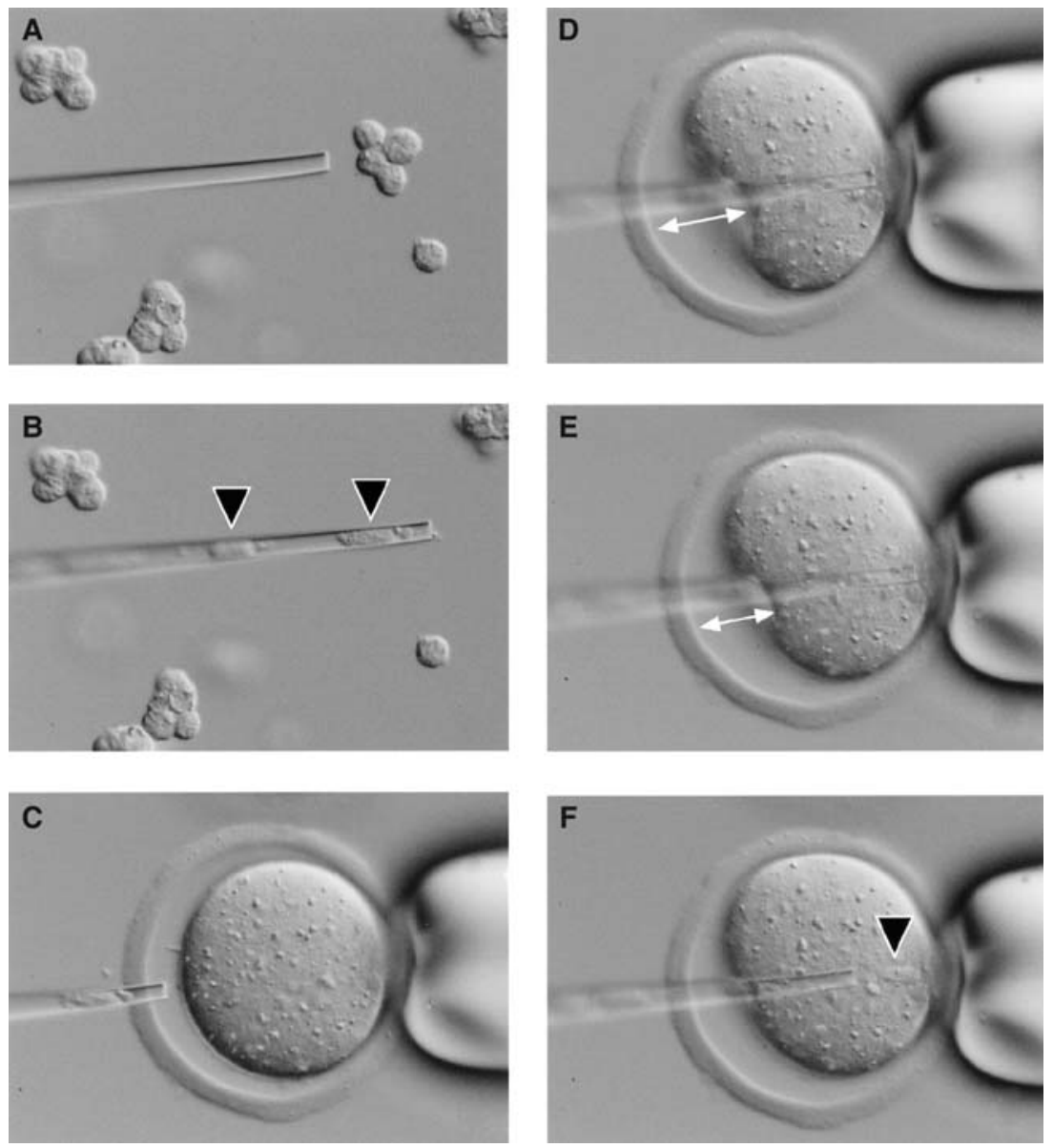

FIGURE 4. Injection of a cumulus cell nucleus into an enucleated oocyte. (A) Dispersed cumulus cells; $(B)$ cumulus cell nuclei (arrowheads) in injection pipette; $(C)$ piercing the zona pellucida with the injection pipette; $(D)$ pushing the injection pipette deep into the enucleated oocyte but not breaking the oolemma. $(E)$ Application of the piezo pulse breaks the oolemma, causing a visual relaxation of the membrane (cf. the lengths of the double arrows in $D$ and $E)$. $(F)$ Injection of the donor cumulus cell nucleus (arrowhead) into the ooplasm. 


\section{Part 6: Activating Embryos and Culturing}

Reconstructed embryos require a signal to activate development. This is induced by culturing the reconstructed embryos in medium containing strontium.

27. Approximately $1 \mathrm{~h}$ after nuclear transfer, activate the oocytes in $\mathrm{Ca}^{2+}$-free $\mathrm{CZB}$ medium containing 2.5-10 mm strontium chloride (CZB-Sr) and $5 \mu \mathrm{g} / \mathrm{mL}$ cytochalasin $\mathrm{B}$ at $37^{\circ} \mathrm{C}$ in $5 \% \mathrm{CO}_{2}$.

The optimal concentration of strontium chloride and time of exposure to the activation medium may vary between laboratories. Contamination of the CZB-Sr medium for activation by $\mathrm{Ca}^{2+}$ carryover from the CZB medium decreases the effect of $\mathrm{Sr}^{2+}$. Therefore, the reconstructed embryos should be washed free of CZB before being activated in CZB-Sr.

The presence of $1 \%$ DMSO in the CZB-Sr activation medium can enhance the reconstructed embryos to develop into blastocysts (Wakayama and Yanagimachi 2001).

28. Approximately $1 \mathrm{~h}$ later, place the activated oocytes in CZB medium containing $5 \mu \mathrm{g} / \mathrm{mL}$ cytochalasin B. Culture for $5 \mathrm{~h}$.

Cytochalasin $B$ is added to the activation medium to prevent extrusion of the donor chromosomes as a polar body. The effects of cytochalasin $B$ and cytochalasin D on cloning experiments do not appear to differ.

29. Wash the reconstructed embryos several times in fresh CZB medium and then culture until the embryos are transferred.

It is important to wash away the cytochalasin thoroughly and quickly because concentrations of cytochalasin $<5 \mu \mathrm{g} / \mathrm{mL}$ often cause deformation of the oocyte surface.

Treatment with TSA, a histone deacetylase inhibitor, for $10 \mathrm{~h}$ following oocyte activation can enhance the reconstructed embryos to develop into term offspring (Kishigami et al. 2006).

30. Roughly assess the success of nuclear transfer by examining the pseudopronuclei of the reconstructed embryos. The embryos should have two or three well-developed pseudopronuclei when they are retrieved from the cytochalasin-containing medium. If none or only one nucleus has formed, the oocytes were activated following nuclear transfer because of inappropriate in vitro handling. Such embryos rarely develop to term (Wakayama and Yanagimachi 2001).

\section{Part 7: Transferring Cloned Embryos}

31. Transfer the cloned embryos into the oviducts or uteri, as in other experiments (see Protocol: Oviduct Transfer [Nagy et al. 2006c] and Protocol: Uterine Transfer [Nagy et al. 2006d], respectively). 
A. Ogura

Special care must be exercised (see Discussion) because cloned embryos are much more sensitive to changes in physical and physiological conditions than are embryos produced by conventional in vitro techniques, such as in vitro fertilization.

\section{DISCUSSION}

ICR female mice have proved to be among the best recipients for cloned embryo transfer. They should be 2.5-4 mo of age and weigh 30-35 g. The use of suboptimal females can result in the loss of entire batches of transferred cloned embryos before implantation (no implantation sites). Thus, ICR females from different vendors should be tested before beginning cloning.

The lower body temperature induced in recipient females by anesthesia may cause poor embryonic development. The use of appropriate heaters helps to maintain body temperature.

Two combinations of embryonic stage and recipient transfer site are used for embryo transfer: (1) Cloned two-cell embryos $(2 \mathrm{~d}$ ) are transferred into the oviducts of 0.5 -dpc pseudopregnant recipient females; and (2) cloned morulae/blastocysts (4 d) are transferred into uteri of 2.5-dpc pseudopregnant recipient females. If low-implantation rates are obtained by transferring cloned two-cell embryos (Day 2), transfer four-cell embryos (Day 3) into the oviducts of 0.5-dpc pseudopregnant recipient females.

\section{RECIPES}

CZB, CZB-HEPES, and CZB-Sr Media for Mouse Cloning

\begin{tabular}{|c|c|c|c|c|c|c|}
\hline & $\begin{array}{c}\mathrm{Emb} \\
(\mathrm{mM})\end{array}$ & $\begin{array}{l}\text { CZB } \\
\text { ryo culture } \\
(\mathrm{mg} / 100 \mathrm{~mL})\end{array}$ & $\begin{array}{r}\mathrm{C} 2 \\
\mathrm{Emb} \\
(\mathrm{mM}) \\
\end{array}$ & $\begin{array}{l}\text { ZB-HEPES } \\
\text { ryo handling } \\
(\mathrm{mg} / 100 \mathrm{~mL})\end{array}$ & $\begin{array}{r}\text { O } \\
(\mathrm{mm}) \\
\end{array}$ & $\begin{array}{l}\mathrm{CZB}-\mathrm{Sr} \\
\text { o activation } \\
(\mathrm{mg} / 100 \mathrm{~mL})\end{array}$ \\
\hline $\mathrm{NaCl}$ & 82.0 & 478.9 & 82.0 & 478.9 & 82.0 & 478.9 \\
\hline $\mathrm{KCl}$ & 4.9 & 36.3 & 4.9 & 36.3 & 4.9 & 36.3 \\
\hline $\mathrm{KH}_{2} \mathrm{PO}_{4}$ & 1.2 & 15.9 & 1.2 & 15.9 & 1.2 & 15.9 \\
\hline $\mathrm{MgSO}_{4} \cdot 7 \mathrm{H}_{2} \mathrm{O}$ & 1.2 & 29.1 & 1.2 & 29.1 & 1.2 & 29.1 \\
\hline $\mathrm{NaHCO}_{3}$ & 25.0 & 210.0 & 15.0 & 126.0 & 25.0 & 210.0 \\
\hline Glucose & 5.6 & 100.0 & 5.6 & 100.0 & 5.6 & 100.0 \\
\hline Sodium pyruvate & 0.3 & 2.9 & 0.3 & 2.9 & 0.3 & 2.9 \\
\hline $\mathrm{CaCl}_{2} \cdot 2 \mathrm{H}_{2} \mathrm{O}^{\mathrm{c}}$ & 1.7 & 25.1 & 1.7 & 25.1 & & \\
\hline $\begin{array}{l}\mathrm{SrCl}_{2} \cdot 6 \mathrm{H}_{2} \mathrm{O}^{\mathrm{c}} \\
\text { HEPES }\end{array}$ & & & 10 & 238.0 & $2.5-10.0$ & $66.7-266.6$ \\
\hline Glutamine $^{c}$ & 1.0 & 14.6 & 1.0 & $\begin{array}{l}20.0 \\
14.6\end{array}$ & 1.0 & 14.6 \\
\hline $\begin{array}{l}\text { Sodium lactate }(60 \% \\
\text { syrup) }\end{array}$ & 20 & $370 \mu \mathrm{L}$ & 20 & $370 \mu \mathrm{L}$ & 20 & $370 \mu \mathrm{L}$ \\
\hline $\begin{array}{l}\text { EDTA } \\
\text { Polyvinyl alcohol } \\
\text { BSA }\end{array}$ & 0.10 & $\begin{array}{c}3.8 \\
10.0 \\
5 \mathrm{mg} / \mathrm{mL}\end{array}$ & 0.10 & $\begin{array}{c}3.8 \\
10.0\end{array}$ & 0.10 & $\begin{array}{c}3.8 \\
10.0 \\
5 \mathrm{mg} / \mathrm{mL}\end{array}$ \\
\hline
\end{tabular}

CZB-based media are used with slight modifications for culturing cloned embryos and micromanipulating oocytes in vitro. These three media should be made every $2 \mathrm{wk}$. Sterilize with Millipore filters $\left(0.45 \mu \mathrm{m}\right.$ ), dispense into 5 - $\mathrm{mL}$ plastic tubes (Falcon 2057), and store at $4^{\circ} \mathrm{C}$ until they are used. Add glutamine and $\mathrm{CaCl}_{2}$ (or $\mathrm{SrCl}_{2}$ ) on the day of the experiment.

${ }^{\mathrm{a}} \mathrm{KSOM}$ medium can also be used successfully to culture cloned mouse embryos.

${ }^{\mathrm{b}}$ The CZB-HEPES micromanipulation medium does not contain bovine serum albumin (BSA), which can decrease the survival rate of oocytes after injection. Bovine calf serum (10\%-20\%) may be used instead of BSA.

${ }^{\mathrm{c}} \mathrm{CaCl}_{2}, \mathrm{SrCl}_{2}$, and glutamine are added from $100 \times, 10 \times$, and $100 \times$ stock solutions, respectively.

${ }^{\mathrm{d}}$ Polyvinyl alcohol (cold-water soluble, e.g., Sigma-Aldrich P8136) is first dissolved in ultrapure water for $1 \mathrm{~h}$ at $80^{\circ} \mathrm{C}$. 
Polyvinylpyrrolidone (PVP; 10\%-12\%; $360 \mathrm{kDa})$

Prepare a $10 \%-12 \%$ PVP $(360 \mathrm{kDa})$ solution in CZB-HEPES medium $<\mathrm{R}>$, and shake overnight to dissolve the PVP completely. Incompletely dissolved PVP solutions may damage the plasma membranes of oocytes and donor cells. The solution can be stored for at least $6 \mathrm{mo}$ at $4^{\circ} \mathrm{C}$ or $-20^{\circ} \mathrm{C}$.

\section{ACKNOWLEDGMENTS}

George Adebayo provided information on using Fluorinert for piezo-driven microcapillaries.

\section{REFERENCES}

Kishigami S, Mizutani E, Ohta H, Hikichi T, Thuan NV, Wakayama S, Bui HT, Wakayama T. 2006. Significant improvement of mouse cloning technique by treatment with Trichostatin A after somatic nuclear transfer. Biochem Biophys Res Commun 340: 183-189.

Lee J, Inoue K, Ono R, Ogonuki N, Kohda T, Kaneko-Ishino T, Ogura A, Ishino F. 2002. Erasing genomic imprinting memory in mouse clone embryos produced from Day 11.5 primordial germ cells. Development 129: $1807-1817$.

McGrath JM, Solter D, Mann J. 2017. Pronuclear transplantation in the mouse embryo. Cold Spring Harb Protoc doi: 10.1101/pdb .prot094417.

Nagy A, Gertsenstein M, Vintersten K, Behringer R. 2006a. Making holding pipettes. Cold Spring Harb Protoc doi: 10.1101/pdb.prot4394.

Nagy A, Gertsenstein M, Vintersten K, Behringer R. 2006b. Collecting zygotes and removing cumulus cells with hyaluronidase. Cold Spring Harb Protoc doi: 10.1101/pdb.prot4358.

Nagy A, Gertsenstein M, Vintersten K, Behringer R. 2006c. Oviduct transfer. Cold Spring Harb Protoc doi: 10.1101/pdb.prot4379
Nagy A, Gertsenstein M, Vintersten K, Behringer R. 2006d. Uterine transfer. Cold Spring Harb Protoc doi: 10.1101/pdb.prot4380.

Ogura A, Inoue K, Ogonuki N, Noguchi A, Takano K, Nagano R, Suzuki O, Lee J, Ishino F, Matsuda J. 2000a. Production of male cloned mice from fresh, cultured, and cryopreserved immature Sertoli cells. Biol Reprod 62: $1579-1584$

Ogura A, Inoue K, Takano K, Wakayama T, Yanagimachi R. 2000b. Birth of mice after nuclear transfer by electrofusion using tail tip cells. Mol Reprod Dev 57: 55-59.

Wakayama T, Yanagimachi R. 2001. Effect of cytokinesis inhibitors, DMSO and the timing of oocyte activation on mouse cloning using cumulus cell nuclei. Reproduction 122: 49-60.

Wakayama T, Perry AC, Zuccotti M, Johnson KR, Yanagimachi R. 1998. Full-term development of mice from enucleated oocytes injected with cumulus cell nuclei. Nature 394: 369-374.

Wakayama T, Rodriguez I, Perry AC, Yanagimachi R, Mombaerts P. 1999. Mice cloned from embryonic stem cells. Proc Natl Acad Sci 96: 14984-14989. 


\section{Cloning Mice}

\section{Atsuo Ogura}

Cold Spring Harb Protoc; doi: 10.1101/pdb.prot094425

\begin{tabular}{rc}
$\begin{array}{r}\text { Email Alerting } \\
\text { Service }\end{array}$ & Receive free email alerts when new articles cite this article - click here. \\
\hline $\begin{array}{c}\text { Subject } \\
\text { Categories }\end{array}$ & $\begin{array}{c}\text { Browse articles on similar topics from Cold Spring Harbor Protocols. } \\
\text { Developmental Biology (728 articles) } \\
\text { Mouse (437 articles) }\end{array}$ \\
& \\
\hline
\end{tabular}

